\title{
Is the Completion Time of a Questionnaire a Pivotal Factor When Proving Feasibility?: Author's Reply
}

TO THE EDITOR: We sincerely appreciate the interest and comments on our paper on the "Comparative study of 2 different questionnaires in Japanese patients: the quality of life and utility evaluation survey technology questionnaire (QUEST) versus the frequency scale for the symptoms of gastroesophageal reflux disease questionnaire (FSSG)." ${ }^{1}$ We do agree with the points raised by the author of the letter on our paper. ${ }^{2}$

Questionnaires are very useful for the diagnosis of diseases, evaluation of patients' conditions, and monitoring of therapeutic responses. However, no questionnaires are widely used in clinical settings for various reasons. In this study, we attempted to identify the reasons for such a circumstance. We thought that a suitable questionnaire requires comprehensibility, simplicity and convenience, in addition to having validity, reliability and responsiveness as a matter of course. These characteristics would require the questionnaires to be filled out by the respondents themselves, without the investigators' aid, within a short period of time. Consequently, we analyzed the time taken by the respondents to complete the questionnaires, what, if any, questions the subjects asked as they filled in the questionnaires, and the questionnaire scores on 2 major diagnostic instruments for GERD being used in Japan.

The time taken for completion of a questionnaire could be considered as a parameter of the feasibility. We demonstrated that the completion time for frequency scale for the symptoms of gastroesophageal reflux disease questionnaire (FSSG) was shorter than for quality of life and utility evaluation survey technology (QUEST) (97.5 [27.0-225.0] vs. 196.5 [54.0-661.0] seconds, respectively; $P<0.0001)$. Actually, we asked the subjects about their impressions of the QUEST and FSSG questionnaires just after they completed both the questionnaires, which revealed that they found most of the items on FSSG easier to answer. In recent years, not only in Korea, but also in Japan, the population of the aged has been increasing. Therefore, questionnaires that can be easily understood by elderly people would be needed in clinical settings. When we reanalyzed our results in only elderly people over the age of 65 years, the difference in the completion time between FSSG and QUEST was even more marked (107.0 [47.0-225.0] vs. 248.0 [126.0-661.0] seconds, respectively; $P<$ $0.0001)$. Furthermore, a significant difference in the completion time for QUEST was found between the younger and older respondents (176.0 [54.0-399.0] vs. 248.0 [126.0-661.0] seconds, respectively; $P=0.004)$. Similarly, the completion time for the FSSG was also significantly different between the younger and elder subjects (84.0 [27.0-186.0] vs. 107.0 [47.0-225.0] seconds, respectively; $P=0.003)$.

There are many limitations to our study. In fact, other factors such as the patients' satisfaction and response rate also need to be taken into account while evaluating the feasibility of a questionnaire. ${ }^{3,4}$ Therefore, our results alone are not sufficient to establish the superiority of FSSG over QUEST. However, this study revealed at least one aspect of the problems involved in the utilization of questionnaires for clinical research. Further investigation of the feasibility of using questionnaires is needed.

\section{Takashi Nonaka and Masahiko Inamori Gastroenterology Division, Yokohama City University Hospital, Yokohama, Japan}

1. Nonaka T, Kessoku T, Ogawa Y, et al. Comparative study of 2 different questionnaires in Japanese patients: the quality of life and utility evaluation survey technology questionnaire (QUEST) versus the frequency scale for the symptoms of gastroesophageal reflux disease questionnaire (FSSG). J Neurogastroenterol Motil 2013;19:54-60.

2. Park JH. Is the completion time of a questionnaire a pivotal factor when proving feasibility? J Neurogastroenterol Motil 2013;19:267.

3. Velanovich V. Comparison of generic (SF-36) vs. disease-specific (GERD-HRQL) quality-of-life scales for gastroesophageal reflux 
disease. J Gastrointest Surg 1998;2:141-145.

4. van Marwijk HW, Wallace P, de Bock GH, et al. Evaluation of the feasibility, reliability and diagnostic value of shortened versions of the geriatric depression scale. Br J Gen Pract 1995;45:195-199.
Conflicts of interest: None. 